MATHEMATICS OF COMPUTATION

Volume 77, Number 261, January 2008, Pages 243-264

S 0025-5718(07)02041-8

Article electronically published on July 26, 2007

\title{
MODIFIED TAYLOR REPRODUCING FORMULAS AND $H$-P CLOUDS
}

\author{
CARLOS ZUPPA
}

\begin{abstract}
We study two different approximations of a multivariate function $f$ by operators of the form $\sum_{i=1}^{N} \mathcal{T}_{r}\left[f, x_{i}\right](x) \mathcal{W}_{i}(x)$, where $\left\{\mathcal{W}_{i}\right\}$ is an $m$ reproducing partition of unity and $\mathcal{T}_{r}\left[f, x_{i}\right](x)$ are modified Taylor polynomials of degree $r$ expanded at $x_{i}$. The first approximation was introduced by Xuli (2003) in the univariate case and generalized for convex domains by Guessab et al. (2005). The second one was introduced by Duarte (1995) and proved in the univariate case. In this paper, we first relax the Guessab's convexity assumption and we prove Duarte's reproduction formula in the multivariate case. Then, we introduce two related reproducing quasi-interpolation operators in Sobolev spaces. A weighted error estimate and Jackson's type inequalities for $h-p$ cloud function spaces are obtained. Last, numerical examples are analyzed to show the approximative power of the method.
\end{abstract}

\section{INTRODUCTION}

Recent developments made in the context of meshless methods have demonstrated the simplicity of adding hierarchical refinements to a low order set of shape functions (e.g. 2, 3, 4). The basic idea of the method is to multiply functions in a partition of unity $\left\{\mathcal{W}_{i}\right\}_{i=1}^{N}$ (i.e. MLSF) by Taylor's polynomials at nodes $x_{i}$. The resulting functions, called $h-p$ cloud shape functions, have good properties, such as high regularity and compactness; linear combinations of these functions can represent polynomials of degree $p$. This property allows the implementation of $p$ and $h-p$ adaptivity leading in many situations to spectral convergence. This combination has also been successfully used with FEM partitions of unity [11].

In this paper, we consider partitions of unity which have algebraic precision equal to $m \geq 1$, and we study quasi-interpolation operators of the form

$$
\mathcal{I S}(x):=\sum_{i=1}^{N} \mathcal{T}_{i}\left[x_{i}, x\right] \mathcal{W}_{i}(x),
$$

where $\mathcal{T}_{i}\left[x_{i}, x\right]$ are modified Taylor polynomials of degree $k$ expanded at $x_{i}$.

It is important to know the algebraic precision that can be obtained with the operator $\mathcal{I S}$. In the univariate case, Xuli proved in 15 that an appropriate combination of Taylor polynomials yields algebraic precision equal to $p=m+k$. This result was generalized by Guessab et al. 8 to the multivariate case when the

Received by the editor August 9, 2005 and, in revised form, October 24, 2006.

2000 Mathematics Subject Classification. Primary 41A17, 41A10; Secondary 65N15, 65N30.

Key words and phrases. Reproducing formulas, $h$ - $p$ clouds method, error estimates.

Partially supported by CyT-FCFMN-UNSL Grant 22/F330. The author wishes to thank the anonymous reviewers for their valuable suggestions. 
domain is convex. Xuli's work, however, was preceded by Duarte. In fact, many years before Xuli, Duarte [2, 3, 4] noted that the use of Taylor polynomials of the same degree as those that are reproduced by $\left\{\mathcal{W}_{i}\right\}$ yield singular or near singular stiffness matrices in Galerkin schemes. Then, he proposed using only polynomials which are missing in the linear combinations of $\left\{\mathcal{W}_{i}\right\}$ and a reproduction formula which he proved only in the univariate case [2].

These different approaches suggest different $h-p$ cloud function spaces. We show in the last section that, amazingly, the first approach produces better numerical results, even if the linear system is ill conditioned. Therefore, further investigation into this approach may be worthwhile.

Our first contribution in this paper deals with reproduction formulas:

- Xuli's reproduction formula: we show that the convexity assumption in 8 can be relaxed. In fact, it is only needed that the support of function $\mathcal{W}_{i}$ be star shaped w.r.t. node $x_{i}, i=1, \ldots, N$.

- Duarte's reproduction formula: we prove it in the multivariate case.

In the second contribution of this work, we introduce two quasi-interpolation operators which are suggested by the reproduction formulas. Averaged Taylor polynomials must be used to define the operators in Sobolev spaces and are a natural generalization of the Verfürth's quasi-interpolation operator [13. Then, we prove a general weighted error estimate for reproducing quasi-interpolation operators. From these error estimates, Jackson's type inequalities can be derived for $h$ - $p$ cloud function spaces.

The paper is organized as follows. In Section 1 we give the basics of the $h-p$ clouds theory. Section 2 deals with the reproduction formulas of Xuli and Duarte. We prove therein the generalization of Guessab's result and Duarte's reproduction formula in the multivariate case. Section 3 is dedicated to quasiinterpolation reproducing operators in Sobolev spaces, error estimates and convergence. Finally, in Section 4, numerical experiments are analyzed to show the approximative power of the $h-p$ associated spaces.

\section{1. $h-p$ CLOUDS}

Let $\Omega$ be an open bounded domain in $\mathbb{R}^{n}$ with Lipschitz boundary $\partial \Omega$. Given $u \in \mathcal{D}^{\prime}(\Omega)$ and $\alpha \in \mathbb{N}_{0}^{n}$ we denote, as usual,

$$
D^{\alpha} u=\left(\frac{\partial}{\partial x_{1}}\right)^{\alpha_{1}} \cdots\left(\frac{\partial}{\partial x_{n}}\right)^{\alpha_{n}} u, \quad|\alpha|=\alpha_{1}+\cdots+\alpha_{n}, \quad \alpha !=\alpha_{1} ! \cdots \alpha_{n} !
$$

For $q \geq 1$ and $s \in \mathbb{N}_{0}$, we call $W_{q}^{s}(\Omega)$ the Sobolev space which consists of all the functions $u \in L^{q}(\Omega)$ such that $D^{\alpha} u \in L^{q}(\Omega)$ for $|\alpha| \leq s$. Given $j \in \mathbb{N}_{0}, 0 \leq j \leq s$, we define

$$
|u|_{j, q}=\left(\sum_{|\alpha|=j}\left\|D^{\alpha} u\right\|_{L^{q}(\Omega)}^{q}\right)^{1 / q}
$$

therefore, the usual norm in $W_{q}^{s}(\Omega)$ is defined by

$$
\|u\|_{s, q}=\left(\sum_{j=0}^{s}|u|_{j, q}^{q}\right)^{1 / q}
$$


If $q=2$, we denote, as usual, $W_{2}^{s}(\Omega)=H^{s}(\Omega)$. When explicit reference to the domain is needed, we denote $\|\cdot\|_{s, q}=\|\cdot\|_{\Omega, s, q}$ and $|\cdot|_{j, q}=|\cdot|_{\Omega, j, q}$.

Let $Q^{N}$ be an arbitrarily chosen set of $N$ points $x_{i} \in \bar{\Omega}$ referred to as nodes

$$
Q^{N}=\left\{x_{1}, x_{2}, \ldots, x_{N}\right\}, \quad x_{i} \in \bar{\Omega},
$$

and let $\mathcal{I}_{N}:=\left\{\omega_{i}\right\}_{i=1}^{N}$ be a finite open covering of $\bar{\Omega}$,

$$
\bigcup_{i=1}^{N} \omega_{i}=\bar{\Omega}
$$

such that $x_{i} \in \omega_{i}, i=1, \ldots, N$.

Remark 1.1. We have assumed $\omega_{i} \subset \bar{\Omega}, \forall i: i=1, \ldots, N$. Then, the sets $\omega_{i}$ are relatively open in $\bar{\Omega}$.

Definition 1.2. Given $d\left(\mathcal{I}_{N}\right), m \in \mathbb{N}$, a class of functions $\mathcal{S}_{N}:=\left\{\mathcal{W}_{i}\right\}_{i=1}^{N}$ will be called a $\left(d\left(\mathcal{I}_{N}\right), m\right)$-partition of unity subordinate to $\mathcal{I}_{N}$ if:

- $\mathcal{W}_{i} \in C(\bar{\Omega}) \cap W_{\infty}^{d\left(\mathcal{I}_{N}\right)}(\Omega), \quad \forall i: i=1, \ldots, N$.

- $\operatorname{supp}\left(\mathcal{W}_{i}\right)=\bar{\omega}_{i}, \quad \forall i: i=1, \ldots, N$.

- $\mathcal{S}_{N}$ has algebraic precision of degree $m$. That is, for every $P \in \mathcal{P}^{m}$, we have

$$
P(x)=\sum_{i=1}^{N} P\left(x_{i}\right) \mathcal{W}_{i}(x), \quad \forall x \in \bar{\Omega},
$$

where $\mathcal{P}^{m}$ denotes the class of all polynomials of degree $\leq m$.

- Every $\omega_{i}$ is star shaped w.r.t. $x_{i}$, that is,

$$
\left[x, x_{i}\right] \subset \omega_{i}, \quad \forall x \in \omega_{i},
$$

where $\left[x, x_{i}\right]$ is the segment joining $x$ and $x_{i}$.

The star shaped condition in (1.2) is a necessary ingredient in the reproducing formulas of the next section. Furthermore, this condition is met in almost all cases of practical interest.

Remark 1.3. If $m>1$, the usual assumption of a partition of unity $\mathcal{W}_{i} \geq 0$ is not valid.

Remark 1.4. For simplicity, from this point on, we assume without explicit mention that we are dealing with a $\left(d\left(\mathcal{I}_{N}\right), m\right)$-partition of unity.

Definition 1.5. For each set $T \subseteq \Omega$, let $i(T)$ be the set of indexes defined by

$$
i(T):=\left\{i: \omega_{i} \bigcap T \neq \emptyset\right\} .
$$

The set

$$
\widehat{T}:=\bigcup_{i \in i(T)} \omega_{i}
$$

is called the super-cloud of $T$.

The diameters

$$
h_{i}:=\sup _{x, y \in \omega_{i}}\|x-y\|
$$

and

$$
d \widehat{T}:=\sup _{x, y \in \widehat{T}}\|x-y\|
$$

are usually key ingredients in error estimates. 
Let $r \geq 0$ be any integer. For $i=1, \ldots, N, \mathcal{P}_{i}^{r}$ denotes the vector space of $r$-Taylor polynomials at $x_{i}$,

$$
\mathcal{P}_{i}^{r}:=\left\{Q: Q(x)=\sum_{0 \leq|\nu| \leq r} a_{\nu}\left(x-x_{i}\right)^{\nu}\right\} .
$$

Definition 1.6. Let $\mathcal{H}$ be some space of functions. Given a linear operator

$$
\mathcal{T}^{t}: \mathcal{H} \rightarrow \prod_{i=1}^{N} \mathcal{P}_{i}^{r}
$$

the associated r-modified approximation operator is the linear operator $\mathcal{S} \mathcal{T}: \mathcal{H} \rightarrow$ $C(\bar{\Omega})$ defined by

$$
\mathcal{S} \mathcal{T}[u]:=\sum_{i=1}^{N} T_{i}^{r}[u] \cdot \mathcal{W}_{i}, \quad u \in \mathcal{H} \text { and } \mathcal{T}^{r}[u]=\left(\mathcal{T}_{i}^{r}[u]\right)_{i=1, \ldots, N} .
$$

In this work, we are mainly interested in the case where $\left(\mathcal{H},\|\cdot\|_{\mathcal{H}}\right)$ is some Sobolev space of functions over $\Omega$ and the approximation operators increase the algebraic precision. That is,

$$
\mathcal{S} \mathcal{T}[P]=P, \quad \forall P \in \mathcal{P}^{m+r}
$$

for some integer $r>0$.

Two modified formulas will be studied in this paper: the first one is based on the works of Xuli [15] and Guessab et al. [8]; the other one refers to the investigation of Duarte and Oden $[3]$.

The $h-p$ approximation operators can be used to estimate the approximating power of $h-p$ cloud shape functions of Duarte and Oden [3].

Definition 1.7. Let $\mathcal{P}_{N}:=\left\{Q_{i}\right\}_{i=1}^{N}$ such that

- $Q_{i}$ is a vectorial subspace of $\mathcal{P}_{i}^{r_{i}}, r_{i} \geq 1, \forall i: i=1, \ldots, N$.

- $Q_{i}$ contains the constant polynomials, $\forall i: i=1, \ldots, N$.

The associated cloud shape function space is the vectorial space defined by

$$
\mathcal{F}\left(\mathcal{P}_{N}\right):=\left\{w \mid w=\sum_{i=1}^{N} P_{i} \mathcal{W}_{i}, P_{i} \in Q_{i}, \forall i: i=1, \ldots, N\right\} .
$$

The finite vectorial space $\mathcal{F}\left(\mathcal{P}_{N}\right)$ can be used in Galerkin approximation of the solution of a boundary value problem. It is well known that a main tool in error estimates for the solution of the boundary value problem is

$$
\inf _{w \in \mathcal{F}\left(\mathcal{P}_{N}\right)}\|u-w\|_{\mathcal{H}}
$$

\section{REPRODUCING FORMULAS}

In this section we study linear operators

$$
\mathcal{T}^{m+r}: C^{\infty}(\bar{\Omega}) \rightarrow \prod_{i=1}^{N} \mathcal{P}_{i}^{m+r}, \quad r \in \mathbb{N}_{0},
$$

such that the associated $(m+r)$-modified approximation operator $\mathcal{S} \mathcal{T}$ has algebraic precision of degree $p=m+r$.

We shall first state an auxiliary result. 
Lemma 2.1. For every multi-index $\beta \in \mathbb{N}_{0}^{n}, 1 \leq|\beta| \leq m$, we have

$$
\sum_{i=1}^{N}\left(x-x_{i}\right)^{\beta}=0, \quad \forall x \in \bar{\Omega} .
$$

Proof. Writing

$$
\left(x-x_{i}\right)^{\beta}=\sum_{0 \leq \delta \leq \beta} \lambda_{\beta, \delta} x^{\beta-\delta} x_{i}^{\delta},
$$

we have

$$
\begin{aligned}
\sum_{i=1}^{N}\left(x-x_{i}\right)^{\beta} \mathcal{W}_{i}(x) & =\sum_{0 \leq \delta \leq \beta} \lambda_{\beta, \delta} x^{\beta-\delta}\left(\sum_{i=1}^{N} x_{i}^{\delta} \mathcal{W}_{i}(x)\right) \\
& =\sum_{0 \leq \delta \leq \beta} \lambda_{\beta, \delta} x^{\beta-\delta} x^{\delta} \\
& =(x-x)^{\beta}=0 .
\end{aligned}
$$

In the univariate case, Xuli [15] discovered that the operator

$$
\mathcal{S} \mathcal{T}[u](x):=\sum_{i=1}^{N} \mathcal{T}_{i}^{m+r}[u](x) \mathcal{W}_{i}(x),
$$

where

$$
\mathcal{T}_{i}^{m+r}[u](x):=\sum_{j=0}^{r} a_{m r j} \frac{u^{(j)}\left(x_{i}\right)}{j !}\left(x-x_{i}\right)^{j}
$$

and

$$
a_{m r j}=\frac{(m+r-j) ! r !}{(m+r) !(r-j) !}
$$

reproduces polynomials up to degree $m+r$.

This result was extended to the multivariate case by Guessab et al. 8 when $\Omega$ is convex. In fact, the convexity of $\Omega$ is a severe restriction that is not needed in the proof. As we shall see, the star shaped condition in (1.2) suffices.

Given numbers $c_{\alpha} \in \mathbb{N}_{0}^{n},|\alpha| \leq r$, we shall consider the linear operators $\mathcal{T}_{i, \mathbf{c}}^{m+r}$ defined by the modified Taylor polynomials

$$
\mathcal{T}_{i, \mathbf{c}}^{m+r}[u](x):=\sum_{|\alpha|=0}^{r} c_{\alpha} D^{\alpha} u\left(x_{i}\right)\left(x-x_{i}\right)^{\alpha} .
$$

For $x \in \bar{\Omega}$, let $\omega_{x}$ be defined by

$$
\omega_{x}:=\left\{i: \mathcal{W}_{i}(x) \neq 0\right\} .
$$

Therefore,

$$
\begin{aligned}
\mathcal{S} \mathcal{T}[u](x) & =\sum_{i=1}^{N} \mathcal{T}_{i, \mathbf{c}}^{m+r}[u](x) \mathcal{W}_{i}(x) \\
& =\sum_{i \in \omega_{x}} \mathcal{T}_{i, \mathbf{c}}^{m+r}[u](x) \mathcal{W}_{i}(x) .
\end{aligned}
$$

It is clear from (1.2) that, if $i \in \omega_{x}$, then $\left[x, x_{i}\right] \subset \omega_{i}$. 
Theorem 2.2. There exist constants $c_{\alpha} \in \mathbb{N}_{0}^{n},|\alpha| \leq r, c_{\alpha}=c(m, r, \alpha)$ with $c_{1}=1$, and constants $\sigma_{\beta} \in \mathbb{N}_{0}^{n},|\beta|=m+r+1, \sigma_{\beta}=\sigma(m, r, \beta)$, such that

$$
\begin{aligned}
u(x)= & \sum_{i \in \omega_{x}} \mathcal{T}_{i, \mathbf{c}}^{m+r}[u](x) \mathcal{W}_{i}(x) \\
& +\sum_{i \in \omega_{x}} R_{i}(x) \mathcal{W}_{i}(x),
\end{aligned}
$$

where

$$
R_{i}(x)=\sum_{|\beta|=m+r+1} \sigma_{\beta}\left(\int_{0}^{1} D^{\beta} u\left(x_{i}+t\left(x-x_{i}\right)\right) d t\right)\left(x-x_{i}\right)^{\beta} .
$$

Proof. We only need to adapt the proof given in 8 . We shall give the details mainly because they will be useful in the analysis of the Duarte and Oden reproducing operator. For $i \in \omega_{x}$, we introduce the univariate function $g_{i}:[0,1] \rightarrow \mathbb{R}$ defined by

$$
g(t):=u\left(x_{i}+t\left(x-x_{i}\right)\right) .
$$

By Taylor's theorem, we have

$$
g_{i}^{(j)}(0)=\sum_{k=j}^{m+r} \frac{(-1)^{k-j}}{(k-j) !} g_{i}^{(k)}(1)-\int_{0}^{1} \frac{(-t)^{m+r-j}}{(m+r-j) !} g_{i}^{(m+r+1)}(t) d t .
$$

Next, we multiply both sides by $a_{m r j} / j$ ! and sum over $j$ from 0 to $r$. Then, we can write

$$
\sum_{j=0}^{r} \frac{a_{m r j}}{j !} \sum_{k=j}^{m+r} \frac{(-1)^{k-j}}{(k-j) !} g_{i}^{(k)}(1)=\sum_{k=0}^{m+r}\left(\sum_{j=0}^{k^{\prime}} \frac{(-1)^{k-j} a_{m r j}}{j !(k-j) !}\right) g_{i}^{(k)}(1),
$$

where $k^{\prime}=\min \{k, r\}$. Furthermore, it was shown in [15] that

$$
\sum_{j=0}^{k^{\prime}} \frac{(-1)^{k-j} a_{m r j}}{j !(k-j) !}=0 \quad(k=m+1, \ldots, m+r) .
$$

Hence, noting that $a_{m r 0}=1$, we obtain

$$
\sum_{j=0}^{r} \frac{a_{m r j}}{j !} \sum_{k=j}^{m+r} \frac{(-1)^{k-j}}{(k-j) !} g_{i}^{(k)}(1)=g_{i}(1)+\sum_{k=1}^{m}\left(\sum_{j=0}^{k^{\prime}} \frac{(-1)^{k-j} a_{m r j}}{j !(k-j) !}\right) g_{i}^{(k)}(1) .
$$

Therefore,

$$
\begin{aligned}
\sum_{j=0}^{r} \frac{a_{m r j}}{j !} g_{i}^{(j)}(0)= & u(x)+\sum_{k=1}^{m}\left(\sum_{j=0}^{k^{\prime}} \frac{(-1)^{k-j} a_{m r j}}{j !(k-j) !}\right) g_{i}^{(k)}(1) \\
& -(-1)^{m} \int_{0}^{1} \frac{t^{m}(1-t)^{r}}{(m+r) !} g_{i}^{(m+r+1)}(t) d t .
\end{aligned}
$$

Finally, we multiply both sides of (2.3) by $\mathcal{W}_{i}(x)$ and sum over $i$ in $\omega_{x}$. Then, it follows that

$$
\sum_{i \in \omega_{x}}\left(\sum_{k=1}^{m}\left(\sum_{j=0}^{k^{\prime}} \frac{(-1)^{k-j} a_{m r j}}{j !(k-j) !}\right) g_{i}^{(k)}(1)\right) \mathcal{W}_{i}(x)
$$


can be rewritten as

$$
\sum_{k=1}^{m}\left(\sum_{j=0}^{k^{\prime}} \frac{(-1)^{k-j} a_{m r j}}{j !(k-j) !}\right)\left(\sum_{i \in \omega_{x}} g_{i}^{(k)}(1) \mathcal{W}_{i}(x)\right) .
$$

Now, it is important to analyze the expression

$$
\sum_{i \in \omega_{x}} g_{i}^{(k)}(1) \mathcal{W}_{i}(x), \quad(k=1, \ldots, m) .
$$

By Leibnitz's rule, it follows that

$$
g_{i}^{(k)}(1)=\sum_{|\beta|=k} \nu_{\beta} D^{\beta} u(x)\left(x-x_{i}\right)^{\beta} .
$$

Hence

$$
\begin{aligned}
\sum_{i \in \omega_{x}} g_{i}^{(k)}(1) \mathcal{W}_{i}(x) & =\sum_{|\beta|=k} \nu_{\beta} D^{\beta} u(x)\left(\sum_{i \in \omega_{x}}\left(x-x_{i}\right)^{\beta} \mathcal{W}_{i}(x)\right) \\
& =\sum_{|\beta|=k} \nu_{\beta} D^{\beta} u(x)\left(\sum_{i=1}^{N}\left(x-x_{i}\right)^{\beta} \mathcal{W}_{i}(x)\right) \\
& =0
\end{aligned}
$$

by (2.1). Finally, we can write

$$
\sum_{i \in \omega_{x}}\left(\sum_{j=0}^{r} \frac{a_{m r j}}{j !} g_{i}^{(j)}(0)\right) \mathcal{W}_{i}(x)=u(x)+\sum_{i \in \omega_{x}} R_{i}(x) \mathcal{W}_{i}(x),
$$

where

$$
R_{i}(x)=-(-1)^{m} \int_{0}^{1} \frac{t^{m}(1-t)^{r}}{(m+r) !} g_{i}^{(m+r+1)}(t) d t,
$$

and the theorem follows.

Corollary 2.3. There exist constants $c_{\alpha} \in \mathbb{N}_{0}^{n}, 1 \leq|\alpha| \leq r, c_{\alpha}=c(m, r, \alpha)$, such that for every $P \in \mathcal{P}^{m+r}$,

$$
\sum_{i=1}^{N}\left(P\left(x_{i}\right)+\sum_{1 \leq|\alpha| \leq r} c_{\alpha} D^{\alpha} P\left(x_{i}\right)\left(x-x_{i}\right)^{\alpha}\right) \mathcal{W}_{i}(x)=P(x), \quad \forall x \in \bar{\Omega} .
$$

Remark 2.4. Constants $c_{\alpha}$ can be effectively calculated and depend on Xuli's constants $a_{m r j}$ and the constants appearing in Leibnitz's rule of composite derivatives.

We observe that the modified Taylor polynomials expanded at nodes $x_{i}$ contain polynomials of low degree that are reproduced by the class $\left\{\mathcal{W}_{i}\right\}$. It was observed by Duarte and Oden [3] that this situation produces singular or nearly singular stiffness matrix in Galerkin approaches because shape functions in the $h-p$ cloud space are not linearly independent. To overcome this drawback, they propose an enrichment that only uses monomials of degree between $m+1$ and $m+r$. The following result was proved by Duarte and Oden in the univariate case and we shall extend it to the multivariate case. 
Theorem 2.5. There exist constants $\widetilde{c}_{\alpha} \in \mathbb{N}_{0}^{n}, m+1 \leq|\alpha| \leq m+r, \widetilde{c}_{\alpha}=c(m, r, \alpha)$, such that for every $P \in \mathcal{P}^{m+r}$,

$$
\sum_{i=1}^{N}\left(P\left(x_{i}\right)+\sum_{m+1 \leq|\alpha| \leq m+r} \widetilde{c}_{\alpha} D^{\alpha} P\left(x_{i}\right)\left(x-x_{i}\right)^{\alpha}\right) \mathcal{W}_{i}(x)=P(x), \quad \forall x \in \bar{\Omega} .
$$

Proof. Setting $u=P$ and proceeding as in the theorem above, we have

$$
g_{i}^{(j)}(0)=\sum_{k=j}^{m+r} \frac{(-1)^{k-j}}{(k-j) !} g_{i}^{(k)}(1) .
$$

We consider first case $j=0$. Then

$$
g_{i}^{(0)}(0)=\sum_{k=0}^{m+r} \frac{(-1)^{k}}{k !} g_{i}^{(k)}(1)
$$

Next, we multiply both sides of (2.6) by a constant $b_{j}$ that must be chosen and we add the sum over $j$ from $m+1$ to $m+r$ to (2.6). That is,

$$
\begin{aligned}
g_{i}^{(0)}(0)+\sum_{j=m+1}^{m+r} b_{j} g_{i}^{(j)}(0)= & \sum_{k=0}^{m+r} \frac{(-1)^{k}}{k !} g_{i}^{(k)}(1) \\
& +\sum_{j=m+1}^{m+r} b_{j}\left(\sum_{k=j}^{m+r} \frac{(-1)^{k-j}}{(k-j) !} g_{i}^{(k)}(1)\right) .
\end{aligned}
$$

Now, we shall show that constants $b_{j}$ can be chosen so that the right term is equal to

$$
\sum_{k=0}^{m} \frac{(-1)^{k}}{k !} g_{i}^{(k)}(1)
$$

that is, we must eliminate all terms $g_{i}^{(k)}(1)$ with $k>m$. These terms can be rewritten as

$$
\sum_{k=m+1}^{m+r}\left(\frac{(-1)^{k}}{k !}+\sum_{j=m+1}^{k} b_{j} \frac{(-1)^{k-j}}{(k-j) !}\right) \text {. }
$$

It is easy to see that constants $b_{j}$ can be determined by induction from $m+1$ to $m+r$. Hence, we can effectively write

$$
g_{i}^{(0)}(0)+\sum_{j=m+1}^{m+r} b_{j} g_{i}^{(j)}(0)=\sum_{k=0}^{m} \frac{(-1)^{k}}{k !} g_{i}^{(k)}(1) .
$$

Next, we multiply both sides of (2.7) by $\mathcal{W}_{i}(x)$ and sum over $i$ from $i$ in $\omega_{x}$ :

$$
\begin{aligned}
\sum_{i \in \omega_{x}}\left(g_{i}^{(0)}(0)+\sum_{j=m+1}^{m+r} b_{j} g_{i}^{(j)}(0)\right) \mathcal{W}_{i}(x)= & P(x) \\
& +\sum_{k=1}^{m} \frac{(-1)^{k}}{k !}\left(\sum_{i \in \omega_{x}} g_{i}^{(k)}(1) \mathcal{W}_{i}(x)\right) .
\end{aligned}
$$


Finally, because of the same reason as before

$$
\sum_{i \in \omega_{x}} g_{i}^{(k)}(1) \mathcal{W}_{i}(x)=0, \quad \forall x \in \bar{\Omega}, k=1, \ldots, m .
$$

Constants $\widetilde{c}_{\alpha}$ are now determined from constants $b_{j}$ and the constants appearing in Leibnitz's rule.

We now have two different operators

$$
\mathcal{T}^{m+r}: C^{\infty}(\bar{\Omega}) \rightarrow \prod_{i=1}^{N} \mathcal{P}_{i}^{m+r}
$$

which can be used in order to construct $(m+r)$-reproducing formulas. We define $\mathcal{T}_{\mathcal{X}, i}^{m+r}$ and $\mathcal{T}_{\mathcal{D}, i}^{m+r}$ by

$$
\mathcal{T}_{\mathcal{X}, i}^{m+r}[u](x):=u\left(x_{i}\right)+\sum_{|\alpha|=1}^{r} c_{\alpha} D^{\alpha} u\left(x_{i}\right)\left(x-x_{i}\right)^{\alpha},
$$

and

$$
\mathcal{T}_{\mathcal{D}, i}^{m+r}[u](x):=u\left(x_{i}\right)+\sum_{|\alpha|=m+1}^{m+r} \widetilde{c}_{\alpha} D^{\alpha} u\left(x_{i}\right)\left(x-x_{i}\right)^{\alpha},
$$

where constants $\left\{c_{\alpha}\right\}$ and $\left\{\widetilde{c}_{\alpha}\right\}$ are determined in (2.2) and (2.5) respectively.

\section{Quasi-Interpolation operators in Sobolev SPACES}

To simplify we shall denote $p=m+r$. In this section we derive from formulas (2.8) and (2.9) two quasi-interpolation operators in the Sobolev space $W_{q}^{p}(\Omega)$ $(1 \leq s<\infty)$ that are $p$-reproducing. These operators are generalizations of those introduced by R. Verfürth in [13. The formulas cannot be applied directly because punctual values do not have the usual meaning for functions in Sobolev spaces. Nonetheless, we can use the Verfürth's projection operators defined in [14.

We shall derive weighted local and global error estimates for these quasi-interpolation operators. In case $m=0$, error estimates for $h$ - $p$ clouds were obtained in [16].

Remark 3.1. Procedures for obtaining error estimates are in general a little cumbersome though standard. We sketch the proofs but some details are omitted. Furthermore, even if the interpolation operators could be defined generally, we shall consider from now on the case $q=2$, which is important in applications. Consequently, several simplifications in notation will clarify the main arguments. In particular, we shall drop the subindexes $q$ on norms and seminorms.

For every $i, i=1, \ldots, N$, we define a projection operator $\mathrm{p}_{p, i}$ of $H^{p}\left(\omega_{i}\right)$ onto $\mathcal{P}_{i}^{p}$ which have the following properties:

$$
\begin{gathered}
D^{\beta}\left(\mathrm{p}_{p, i} u\right)=\mathrm{p}_{p-j, i}\left(D^{\beta} u\right), \\
\int_{\omega_{i}} D^{\beta}\left(u-\mathrm{p}_{p, i} u\right)=0
\end{gathered}
$$


for all $u \in H^{p}\left(\omega_{i}\right)$, all $0 \leq j \leq p$, and all $\beta \in \mathbb{N}_{0}^{n}$ with $|\beta|=j$. To this end, we shall follow [14. We denote by

$$
\pi_{i} u:=\frac{1}{\left|\omega_{i}\right|} \int_{\omega_{i}} u
$$

the mean value. For any $u \in H^{p}\left(\omega_{i}\right)$, polynomials $P_{s, i}(u), \ldots, P_{0, i}(u)$ are defined recursively by

$$
P_{s, i}(u):=\sum_{|\alpha|=s} \frac{1}{\alpha !}\left(x-x_{i}\right)^{\alpha} \pi_{i}\left[D^{\alpha} u\right],
$$

and, for $k=p, p-1, \ldots, 1$,

$$
P_{p-1, i}(u):=P_{p, i}(u)+\sum_{|\alpha|=k-1} \frac{1}{\alpha !}\left(x-x_{i}\right)^{\alpha} \pi_{i}\left[D^{\alpha}\left(u-P_{k, i}(u)\right)\right] .
$$

Finally, we set

$$
\mathrm{p}_{p, i}:=P_{0, i} .
$$

Properties (3.1) and (3.2) are proved in [14. Furthermore, it is easy to see that

$$
\mathrm{p}_{p, i}(P)=P, \quad \forall P \in \mathcal{P}^{p} .
$$

Proposition 3.2. There exist constants $C_{\alpha, \beta}=C(\alpha, \beta),|\alpha|<|\beta| \leq s$, such that, for all $i=1, \ldots, N$, if

$$
\mathrm{p}_{p, i}[u](x)=\sum_{\alpha} a_{i, \alpha}[u]\left(x-x_{i}\right)^{\alpha},
$$

then

$$
\left|a_{i, \alpha}[u]\right| \leq\left|\omega_{i}\right|^{-1 / 2}\left(\frac{1}{\alpha !}|| D^{\alpha} u \|_{\omega_{i}, 0}+\left.\sum_{|\alpha|<|\beta| \leq p} C_{\alpha, \beta} h_{i}^{|\beta|-|\alpha|}|| D^{\beta} u\right|_{\omega_{i}, 0}\right),
$$

for all $u \in H^{p}\left(\omega_{i}\right)$.

Proof. Using

$$
\left|\pi_{i}\left[\left(x-x_{i}\right)^{\delta}\right]\right| \leq h_{i}^{|\delta|}
$$

and

$$
\left|\pi_{i}\left[D^{\gamma} u\right]\right| \leq \frac{1}{\left|\omega_{i}\right|}\left(|| D^{\gamma} u\left\|_{\omega_{i}, 0}\right\| \chi_{\omega_{i}} \|_{\omega_{i}, 0}\right)=\left|\omega_{i}\right|^{-1 / 2}|| D^{\gamma} u \|_{\omega_{i}, 0},
$$

the result follows in a standard way by induction from (3.3) and (3.4).

Definition 3.3. We now define the $h-p$ cloud function spaces $\mathcal{F}_{\mathcal{X}}^{m, r}:=\mathcal{F}\left(\left\{\mathcal{P}_{i}^{r}\right\}_{i=1}^{N}\right)$ and $\mathcal{F}_{\mathcal{D}}^{m, r}:=\mathcal{F}\left(\left\{Q_{i}\right\}_{i=1}^{N}\right)$, where

$$
\begin{gathered}
Q_{i}:=\mathbb{R} \oplus \mathcal{P}_{i}^{m+1, m+r}, \\
\mathcal{P}_{i}^{m+1, m+r}:=\left\{S: S=\sum_{m+1 \leq|\beta| \leq m+r} a_{\beta}\left(x-x_{i}\right)^{\beta}\right\}
\end{gathered}
$$

and linear operators $\mathcal{S} \mathcal{T}_{\mathcal{X}}: H^{p}(\Omega) \rightarrow \mathcal{F}_{\mathcal{X}}^{m, r}$ and $\mathcal{S} \mathcal{T}_{\mathcal{D}}: H^{p}(\Omega) \rightarrow \mathcal{F}_{\mathcal{D}}^{m, r}$ by

$$
\mathcal{S} \mathcal{T}_{\mathcal{X}}[u]:=\sum_{i=1}^{N} \mathcal{T}_{\mathcal{X}, i}^{s}\left[p_{s, i}(u)\right] \mathcal{W}_{i}
$$


and

$$
\mathcal{S T}_{\mathcal{D}}[u]:=\sum_{i=1}^{N} \mathcal{T}_{\mathcal{D}, i}^{s}\left[p_{s, i}(u)\right] \mathcal{W}_{i}
$$

respectively, for $u \in H^{p}(\Omega)$.

Using (2.2), (2.5) and the properties of $\mathrm{p}_{p, i}$, it follows easily that $\mathcal{S} \mathcal{T}_{\mathcal{X}}$ and $\mathcal{S} \mathcal{T}_{\mathcal{D}}$ are $p$-reproducing.

Proposition 3.4. $S \mathcal{T}_{\mathcal{X}}$ and $\mathcal{S} \mathcal{T}_{\mathcal{D}}$ have algebraic precision equal to $p$.

Error estimates for two quasi-interpolation linear operators will be considered later in this section. From now on, we shall only consider operator $\mathcal{S} \mathcal{T}_{\mathcal{X}}$. Similar results, however, can be stated for $\mathcal{S T}_{\mathcal{D}}$.

In what follows, the following estimate is needed.

Proposition 3.5. There exist constants $C_{\alpha, \beta}=C(s, \alpha, \beta), 0 \leq|\beta| \leq|\alpha| \leq s$, such that, for all $i=1, \ldots, N$, and all $u \in H^{p}(\Omega)$, we have

$$
\left\|D^{\beta}\left(\mathcal{T}_{\mathcal{X}, i}^{S}\left[p_{s, i}(u)\right]\right)\right\|_{\omega_{i}, 0} \leq\left|\omega_{i}\right|^{-1 / 2} \sum_{|\beta| \leq|\alpha| \leq s} C_{\alpha, \beta} h_{i}^{|\alpha|-|\beta|}\left\|D^{\alpha} u\right\|_{\omega_{i}, 0} .
$$

Proof. (3.5) can be deduced from (3.2), taking into account the constants that modify Taylor polynomials in (2.2) and (2.5).

Given $u \in H^{p}(\Omega)$, note that $\mathcal{S} \mathcal{T}_{\mathcal{X}}[u]$ belong to $H^{\varkappa}(\Omega)$ where $\varkappa:=\min \left(p, d\left(\mathcal{I}_{N}\right)\right)$ (remember that $d\left(\mathcal{I}_{N}\right)$ is the class of weak differentiability of functions $\mathcal{W}_{i}$.

We shall now be concerned with estimates of

$$
\left\|u-\mathcal{S T}_{\mathcal{X}}[u]\right\|_{k}, \quad 0 \leq k \leq \varkappa, \quad u \in H^{p+1}(\Omega) .
$$

The usual ingredients in error estimates, which are assumed from now on, are:

A1. Constants $D_{j, \alpha}, j=1, \ldots, N$ and $0 \leq|\alpha| \leq d_{\mathcal{I}_{N}}$, such that

$$
\left\|D^{\alpha} \mathcal{W}_{j}\right\|_{L^{\infty}\left(\omega_{i}\right)} \leq \frac{D_{j, \alpha}}{h_{j}^{|\alpha|}}
$$

Hence,

$$
\left\|D^{\alpha} \mathcal{W}_{j}\right\|_{\omega_{i}, 0} \leq \frac{D_{j, \alpha}}{h_{j}^{|\alpha|}}\left|\omega_{j}\right|^{1 / 2}
$$

A2. Approximation on super-clouds: there exist constants $B_{i, k}, i=1, \ldots, N$, such that for every $u \in H^{p+1}\left(\widehat{\omega}_{i}\right)$, there exists a polynomial $P_{i}[u]$ of degree $p$ which satisfies

$$
\| u-\left.P_{i}[u]\right|_{\widehat{\omega}_{i}, k} \leq B_{i, k} d_{i}^{p+1-k}|u|_{\widehat{\omega}_{i}, p+1}, \quad k=0, \ldots, p .
$$

Given a fixed $i$, we set $R_{i}:=u-P_{i}[u]$. Then

$$
\mathcal{S} \mathcal{T}_{\mathcal{X}}[u]=P_{i}+\mathcal{S} \mathcal{T}_{\mathcal{X}}\left[R_{i}\right]
$$

and

We can write

$$
u-\mathcal{S} \mathcal{T}_{\mathcal{X}}[u]=R_{i}-\mathcal{S} \mathcal{T}_{\mathcal{X}}\left[R_{i}\right]
$$

$$
\left\|u-\mathcal{S T}_{\mathcal{X}}[u]\right\|_{\omega_{i}, k} \leq\left\|R_{i}\right\|_{\omega_{i}, k}+\left\|\mathcal{S} \mathcal{T}_{\mathcal{X}}\left[R_{i}\right]\right\|_{\omega_{i}, k} .
$$


By (3.7) we have

$$
\left\|R_{i}\right\|_{\omega_{i}, k} \leq\left\|R_{i}\right\|_{\widehat{\omega}_{i}, k} \leq B_{i, k} d_{i}^{p+1-k}|u|_{\widehat{\omega}_{i}, s+1}, \quad k=0, \ldots, p .
$$

Now, we shall try to estimate $\left\|\mathcal{S} \mathcal{T}_{\mathcal{X}}\left[R_{i}\right]\right\|_{\omega_{i}, k}$ for $k=0, \ldots, \varkappa$.

In $\omega_{i}$ we can write

$$
\mathcal{S} \mathcal{T}_{\mathcal{X}}\left[R_{i}\right]=\sum_{j \in \hat{i}} \mathcal{T}_{\mathcal{X}, j}^{p}\left[p_{p, j}\left(R_{i}\right)\right] \mathcal{W}_{j}
$$

Hence, for $\beta, 0 \leq|\beta| \leq \varkappa$, we have

$$
\left\|D^{\beta}\left(\mathcal{S} \mathcal{T}_{\mathcal{X}}\left[R_{i}\right]\right)\right\|_{\omega_{i}, 0} \leq \sum_{j \in \hat{i}}\left\|D^{\beta}\left(\mathcal{T}_{\mathcal{X}, j}^{p}\left[p_{p, j}\left(R_{i}\right)\right] \mathcal{W}_{j}\right)\right\|_{\omega_{j}, 0} .
$$

Remark 3.6. In what follows we shall need to use the expression $\mathcal{T}_{\mathcal{X}, j}^{p}\left[p_{s, j}\left(R_{i}\right)\right]$ at several stages. Then, to simplify, we set

$$
\widetilde{R_{j, i}}:=\mathcal{T}_{\mathcal{X}, j}^{p}\left[p_{p, j}\left(R_{i}\right)\right]
$$

For $j \in \widehat{i}$, by Leibnitz's rule we have

$$
D^{\beta}\left(\widetilde{R_{j, i}} \mathcal{W}_{j}\right)=\sum_{\gamma+\delta=\beta} C(\beta, \gamma, \delta) D^{\gamma} \widetilde{R_{j, i}} D^{\delta} \mathcal{W}_{j}
$$

Therefore,

$$
\left\|D^{\beta}\left(\widetilde{R_{j, i}} \mathcal{W}_{j}\right)\right\|_{\omega_{j}, 0} \leq \sum_{\gamma+\delta=\beta} C(\beta, \gamma, \delta)\left\|D^{\gamma} \widetilde{R_{j, i}}\right\|_{\omega_{j}, 0}\left\|D^{\delta} \mathcal{W}_{j}\right\|_{\omega_{j}, 0} .
$$

On the other hand, recalling (3.5) and (3.6), we get

$$
\left\|D^{\gamma} \widetilde{R_{j, i}}\right\|_{\omega_{j}, 0} \leq\left|\omega_{j}\right|^{-1 / 2} \sum_{|\gamma| \leq|\alpha| \leq \varkappa} C_{\alpha, \beta} h_{j}^{|\alpha|-|\gamma|}\left\|D^{\alpha} R_{i}\right\|_{\omega_{j}, 0}
$$

and

Then,

$$
\left\|D^{\delta} \mathcal{W}_{j}\right\|_{\omega_{j}, 0} \leq \frac{D_{j, \delta}}{h_{j}^{|\delta|}}\left|\omega_{j}\right|^{1 / 2}
$$

$$
\begin{aligned}
\left\|\left.D^{\gamma} \widetilde{R_{j, i}}\right|_{\omega_{j}, 0}|| D^{\delta} \mathcal{W}_{j}\right\|_{\omega_{j}, 0} & \leq D_{j, \delta} \sum_{|\gamma| \leq|\alpha| \leq \varkappa} C_{\alpha, \beta} h_{j}^{|\alpha|-|\beta|}\left\|D^{\alpha} R_{i}\right\|_{\omega_{j}, 0} \\
& \leq D_{j, \delta} \sum_{|\gamma| \leq|\alpha| \leq \varkappa} C_{\alpha, \beta} h_{j}^{|\alpha|-|\beta|}\left\|D^{\alpha} R_{i}\right\|_{\widehat{\omega}_{j}, 0} .
\end{aligned}
$$

Hence,

$$
\left\|\left.D^{\gamma} \widetilde{R_{j, i}}\right|_{\omega_{j}, 0}|| D^{\delta} \mathcal{W}_{j}\right\|_{\omega_{j}, 0} \leq C_{j, \delta, \gamma, i} h_{j}^{|\alpha|-|\beta|} d_{i}^{p+1-|\alpha|}|u|_{\widehat{\omega}_{i}, p+1},
$$

where

$$
C_{j, \delta, \gamma, i}:=D_{j, \delta} \sum_{|\gamma| \leq|\alpha| \leq s} C_{\alpha, \beta} B_{i,|\alpha|} .
$$

Since $h_{j} \leq d_{i}$, we obtain

$$
\left.\left.|| D^{\gamma} \widetilde{R_{j, i}}\right|_{\omega_{j}, 0}|| D^{\delta} \mathcal{W}_{j}\right|_{\omega_{j}, 0} \leq C_{j, \delta, \gamma, i} d_{i}^{p+1-|\beta|}|u|_{\widehat{\omega}_{i}, p+1} .
$$

Now inserting (3.11) into (3.9), we get

$$
\left\|D^{\beta}\left(\widetilde{R_{j, i}} \mathcal{W}_{j}\right)\right\|_{\omega_{j}, 0} \leq \widetilde{C}_{i, j, \beta} d_{i}^{s+1-|\beta|}|u|_{\widehat{\omega}_{i}, p+1},
$$


where

$$
\widetilde{C}_{i, j, \beta}=C\left(D_{j, \delta}, B_{i, k}, n, s, \beta, c_{\rho}\right) .
$$

Finally, summing up over $j$ and adding the bounds in (3.8), we have proved the following local error estimate:

Theorem 3.7. Assume $A 1$ and A2. Then, for every $i, i=1, \ldots, N$, there exist constants $\bar{C}_{i, k}=C_{i, k}\left(D_{j, \delta}, B_{i, k}, n, s, \beta, c_{\rho}\right)$, such that

$$
\left\|u-\mathcal{S} \mathcal{T}_{\mathcal{X}}[u]\right\|_{\omega_{i}, k} \leq \bar{C}_{i, k} d_{i}^{p+1-k}|u|_{\widehat{\omega}_{i}, p+1},
$$

for all $k=0, \ldots, \varkappa$ and all $u \in H^{p+1}(\Omega)$.

Inequality (3.11) can be written in a different way if we assume:

A3. For every $i, i=1, \ldots, N$, there are constants $A_{i}, \bar{A}_{i}$, such that

$$
A_{i} d_{i} \leq h_{j} \leq \bar{A}_{i} d_{i}, \quad \forall j \in i\left(\omega_{i}\right) \text {. }
$$

Using $A_{i}, \bar{A}_{i}$, 3.10 becomes

$$
\left\|\left.D^{\gamma} \widetilde{R_{j, i}}\right|_{\omega_{j}, 0}|| D^{\delta} \mathcal{W}_{j}\right\|_{\omega_{j}, 0} \leq C_{j, \delta, \gamma, \beta} h_{j}^{p+1-|\beta|}|u|_{\widehat{\omega}_{i}, s+1},
$$

where

$$
C_{j, \delta, \gamma, \beta}:=D_{j, \delta} \sum_{|\gamma| \leq|\alpha| \leq s} C_{\alpha, \beta} B_{i,|\alpha|}\left(A_{i}^{-(p+1-|\alpha|)}\right),
$$

and, instead of (3.12), we now get

$$
\|\left. D^{\beta}\left(\widetilde{R_{j, i}} \mathcal{W}_{j}\right)\right|_{\omega_{j}, 0} \leq \widetilde{C}_{i, j, \beta} h_{j}^{p+1-|\beta|}|u|_{\widehat{\omega}_{i}, p+1} .
$$

The local estimate can be written in a weighted form. In fact, let

$$
\left\{\widetilde{C}_{i, \beta}\right\}, \quad 0 \leq|\beta| \leq s ; i=1, \ldots, N
$$

be constants such that

$$
\begin{aligned}
\int_{\omega_{i}}\left|D^{\beta}\left(u-\mathcal{S} \mathcal{T}_{\mathcal{X}}[u]\right)\right|^{p} & \leq \widetilde{C}_{i, \beta}^{p} d_{i}^{(s+1-|\beta|) p}|u|_{\widehat{\omega}_{i}, s+1}^{p} \\
& \leq \widetilde{C}_{i, \beta}^{p} d_{i}^{(s+1-|\beta|) p}\left(\sum_{j \in \widehat{i}}|u|_{\omega_{j}, s+1}^{p}\right) .
\end{aligned}
$$

Now we define functions $g_{i, \beta}:=\widetilde{C}_{i, \beta} d_{i}^{(s+1-|\beta|)} \chi_{\omega_{i}}$ and $g_{\beta}:=\max _{i=1, \ldots, N}\left\{g_{i, \beta}\right\}$. Then, it follows easily that

$$
\int_{\omega_{i}}\left|D^{\beta}\left(u-\mathcal{S} \mathcal{T}_{\mathcal{X}}[u]\right)\right|^{p} \leq \sum_{j \in \hat{i}}\left(\sum_{|\alpha|=s+1} \int_{\omega_{j}} g_{\beta}^{p}\left|D^{\alpha} u\right|^{p}\right) .
$$

The local estimate and a measure of the overlap of clouds,

$$
M=\sup _{i=1, \ldots, N}\{\# \widehat{i}\}
$$

where $\# S$ denotes the number of elements in a finite set $S$, are cornerstones in obtaining weighted global error estimates. In fact, by Lemma 3.3 of [16] and (3.14), we get 
Corollary 3.8. Assume A1 and A2. Then, for all $\beta \in \mathbb{N}_{0}^{n}, 0 \leq|\beta| \leq s$, we have

$$
\left\|D^{\beta}\left(u-\mathcal{S T}_{\mathcal{X}}[u]\right)\right\|_{\Omega, 0} \leq M\left(\sum_{|\alpha|=s+1} \int_{\Omega} g_{\beta}^{2}\left|D^{\alpha} u\right|^{2}\right)^{1 / 2}
$$

for all $u \in H^{p+1}(\Omega)$.

If $d:=\max _{i=1, \ldots, N}$, it is nowadays standard, using the results above, to obtain error estimates in the form

$$
\left\|u-\mathcal{S T}_{\mathcal{X}}[u]\right\|_{k} \leq C_{k} d^{s+1-r}|u|_{s+1}, \quad \forall u \in H^{p+1}(\Omega), \quad k=0, \ldots, \varkappa
$$

and, as a corollary, Jackson type inequalities

$$
\sup _{u \in H^{p+1}(\Omega)} \inf _{w \in \mathcal{F}_{\mathcal{X}}^{m, r}} \frac{|u-w|_{k}}{|u|_{p+1}} \leq C_{k} d^{p+1-k}, \quad k=0, \ldots, \varkappa
$$

if uniform bounds are assumed for all constants involved.

In a similar way, we can obtain

$$
\sup _{u \in H^{p+1}(\Omega)} \inf _{w \in \mathcal{F}_{\mathcal{D}}^{m, r}} \frac{|u-w|_{k}}{|u|_{p+1}} \leq C_{k} d^{p+1-k}, \quad k=0, \ldots, \varkappa .
$$

3.1. FEM partitions of unity. A convergence result. Up to now, we have seen that the key tools in order to obtain error estimates for the interpolation operators are the usual ones:

- A control over the derivatives of the basis functions $\left\{\mathcal{W}_{i}\right\}$.

- A control of the overlapping of the clouds.

- The Poincaré constants involved in local approximation by polynomials.

For general partition of unity, it is not clear how these ingredients can be controlled by simple geometrical properties.

We shall now discuss this problem and convergence results for the simplest case of FEM partitions of unity (see [11]). Therefore, we assume that $\Omega$ is a polyhedral domain and let $\left\{\mathcal{T}_{h}\right\}$ be a family of conforming triangulations of $\bar{\Omega}$. Let $h$ stand for the mesh-size; namely $h:=\max _{T \in \mathcal{T}_{h}} h_{T}$, with $h_{T}$ being the diameter of the simplex $T$. For each $\mathcal{T}_{h}$ we denote with $V_{h} \subset H^{1}(\Omega)$ the standard finite element space of continuous piecewise linear elements and $\left\{\mathcal{W}_{h, i}\right\}$ are the standard shape functions at the nodes $x_{i}$ of the triangulations $\mathcal{T}_{h}$. Here, of course, $d_{\mathcal{I}_{N}}=1$. The discussion that follows is valid, however, for FEM partitions of unity of higher order algebraic precision.

We assume that the meshes $\left\{\mathcal{T}_{h}\right\}$ satisfy some regularity condition. For example, we may assume the following weak regularity assumptions:

Condition 3.9. For $K \geq 1$ and $\epsilon>0$, the mesh $\mathcal{T}_{h}$ satisfies

$$
|T| \geq \epsilon h_{T}^{n}, \quad \text { for all } T \in \mathcal{T}_{h},
$$

and

$$
h_{T} \leq K h_{S}, \quad \text { for all neighboring elements } T, S \in \mathcal{T}_{h} .
$$

Then, it is clear that the first two items can be uniformly controlled independently of $\mathcal{T}_{h}$. We shall discuss the third one. 
First, we introduce some notation. For $T \in \mathcal{T}_{h}$ we define $\operatorname{Ad}(T):=\left\{S \in \mathcal{T}_{h}\right.$ : $S \cap T \neq \emptyset\}$,

$$
\widehat{T}:=\bigcup_{S \in \operatorname{Ad}(T)} S=\bigcup_{x_{i} \in T} \bar{\omega}_{i}
$$

and we denote by $\left\{c_{\widehat{T}, p}\right\}$ the set of Poincare constants associated to $\widehat{T}$. That is, the constants appearing in approximating functions $u \in H^{p+1}(\widehat{T})$ by polynomials of degree $p$ as in (3).

With exactly the same proof, Theorem 3.7 can now be stated in the following form:

Theorem 3.10. For every simplex $T \in \mathcal{T}_{h}$, there exist constants

$$
\bar{C}_{T, k}=C_{T, k}\left(K, \epsilon, c_{\widehat{T}, p}\right)
$$

such that

$$
\left\|u-\mathcal{S} \mathcal{T}_{\mathcal{X}}[u]\right\|_{T, k} \leq \bar{C}_{T, k}\left(\sum_{S \in \operatorname{Ad}(T)} h_{S}^{p+1-k}|u|_{S_{i}, p+1}\right),
$$

for $k=0,1$ and all $u \in H^{p+1}(\widehat{T})$.

The necessity of using constants $\left\{c_{\widehat{T}, p}\right\}$ over all $\widehat{T}$ is somewhat unsatisfactory and it is a fault of our proof. It would be better if we could obtain an estimate that uses constants $\left\{c_{\omega_{j}, p}\right\}$ for $x_{j} \in T$. The clouds $\omega_{j}$ are star-shaped and constants $\left\{c_{\omega_{j}, p}\right\}$ can be obtained by Verfürth's formula [13, 14. In contraposition, $\widehat{T}$ could be non-star-shaped. Anyway, even if the bounds may not be the optimal one, it is true that constants $\left\{c_{\widehat{T}, p}\right\}$ can be uniformly bounded by constants depending on $K, \epsilon$.

Now we shall see the main arguments for justifying this assertion.

The best estimates of Poincaré's constants which are known to us for star shaped domains are due to Verfürth [14] and Durán [5]. Verfürth's bounds do not depend on eccentricity in the case of convex sets. Moreover, for non-convex domains with a re-entrant corner, the bounds are uniform w.r.t. the exterior angle. In order to state Verfürth's results for non-convex but star-shaped domains $U$, we need to state some more definitions. For $z \in U$, we define

$$
\chi(z):=\max _{y \in \partial U}\|y-z\| / \min _{y \in \partial U}\|y-z\| .
$$

Now, assume that $U$ is non-convex but star-shaped w.r.t. at least one point and let $\mathcal{S}_{U}:=\{z \in U: U$ is star-shaped w.r.t. $z\}$. It is clear that there exists a point $z_{U} \in U$, such that $\chi\left(z_{U}\right)=\min _{z \in \mathcal{S}}\{\chi(z)\}$. Then, the number $\mu$ is defined by

$$
\mu_{U}:=\chi\left(z_{U}\right) \text {. }
$$

The main Verfürth's result in 14 is:

Theorem 3.11. Let $U$ be a domain star-shaped w.r.t. at least one point. For $p \in$ $\mathbb{N}_{0}$, there exist constants $c_{p, j}, 0 \leq j \leq m$, such that

$$
\left\|u-Q_{B_{U}}^{m} u\right\|_{j} \leq c_{p, j} d_{U}^{p+1-j}|u|_{p+1}, \quad \forall u \in H^{p+1}(U),
$$

where $d_{U}$ is the diameter of $U$. When $U$ is a convex domain, $B_{U}=U$ and $c_{p, j}=$ $c_{p, j}(n, p)$, i.e., the bounds $c_{p, j}$ depend only on $n$ and $p$. In the non-convex case, $B_{U}=B\left(z_{U}, \varrho\right), \varrho=\operatorname{dist}\left(z_{U}, \partial U\right)$, and $c_{p, j}=c_{p, j}\left(n, p, \mu_{U}\right)$. 
We can obtain uniform estimates for Poincaré's constants of clouds $\omega_{i}$ for any partition $\mathcal{T}_{h}$. In fact, the regularity (3.9) enables us to obtain two constants $\bar{a}(K, \epsilon), \bar{b}(K, \epsilon)$ such that

$$
\bar{a}(K, \epsilon) \leq \mu_{\omega_{i}} \leq \bar{b}(K, \epsilon)
$$

for all $\omega_{i}$ and all $\mathcal{T}_{h}$. Hence, by using (3.11) we obtain the uniform estimates

$$
a(K, \epsilon) \leq c_{\omega_{i, k}} \leq b(K, \epsilon), \quad k=0, \ldots, p
$$

for all $\omega_{i}$ and all $\mathcal{T}_{h}$.

Now, let $T \in \mathcal{T}_{h}$ and $\rho_{T}$ the diameter of the maximal ball $B_{T}$ included in $T$. Using the regularity (3.9) again, we can see that there exist two constants $c(K, \epsilon), c(K, \epsilon)$, such that

$$
c(K, \epsilon) \rho_{T} \leq d_{\widehat{T}} \leq d(K, \epsilon) \rho_{T}
$$

for all $T \in \mathcal{T}_{h}$ and all $\mathcal{T}_{h}$.

If $\widehat{T}$ is star-shaped, the Poincaré's constants can be estimated by Verfürth's result and the regularity of the meshes.

The following result, which relates the Sobolev norm of polynomials at different balls can be easily obtained.

Proposition 3.12. For each $p \in \mathbb{N}_{0}$ there exist two constants $\alpha=\alpha\left(\rho_{T}, d_{T}, p\right), \beta=$ $\beta\left(\rho_{T}, d_{T}, p\right)$ such that

$$
\alpha\|P\|_{B_{T}, p} \leq\|P\|_{\widehat{T}, p} \leq \beta\|P\|_{B_{T}, p}, \quad \forall P \in \mathcal{P}^{p} .
$$

As $\widehat{T}=\bigcup_{x_{i} \in T} \omega_{i}$ and $B_{T} \subset \omega_{i} \cap \omega_{j}$ if $x_{i}, x_{j} \in T$, we can use the arguments of Theorem 7.1 in [6], (3.18) and (3.20) to obtain

Theorem 3.13. There exists a constant $C=C(K, \epsilon, p)$ such that

$$
c_{\widehat{T}, k} \leq C, \quad k=0, \ldots, p,
$$

for all $T \in \mathcal{T}_{h}$.

As a consequence, we finally obtain

Corollary 3.14. There exists a constant $M=M(K, \epsilon, \nu)$, where $\nu:=\#\left\{x_{i} \in T\right\}$ such that

$$
\left\|u-\mathcal{S T}_{\mathcal{X}}[u]\right\|_{\Omega, k} \leq M\left(\sum_{T \in \mathcal{T}_{h}} h_{T}^{p+1-k}|u|_{T, p+1}\right)
$$

for all $u \in H^{p+1}(\Omega)$ and $k=0, \ldots, \varkappa$.

This results grants the $h$-convergence of the interpolator $\mathcal{S} \mathcal{T}_{\mathcal{X}}$ under the simplest geometrical condition of FEM theory.

Remark 3.15. In order to study $p$-convergence, it is clearly necessary to know the asymptotic behavior of Poincaré's constant; but this is beyond the scope of this work. 


\section{Boundary value problems}

Taking into account the Jackson type inequalities (3.15) and $(3.16)$, spaces $\mathcal{F}_{\mathcal{X}}^{m, r}$ and $\mathcal{F}_{\mathcal{D}}^{m, r}$ could be used in Galerkin schemes to solve numerically boundary value problems (see [3, 11]).

We have remarked that, if $m \geq 1$ as is the case when the $h$ - $p$ cloud space is over a FEM partition of unity [11, $\overline{\mathcal{F}}_{\mathcal{X}}^{m, r}$ yields a singular or almost singular stiffness matrix. That is why Duarte and Oden introduce the space $\mathcal{F}_{\mathcal{D}}^{m, r}$. There are, however, several reasons for the interest in $\mathcal{F}_{\mathcal{X}}^{m, r}$ :

- The Generalized Finite Element Method can also yield a sparse positive semi-definite linear system. However, in [12, the use of direct solvers like subroutines MA27 and MA47 of the Harwell Subroutine Library was successful even when the nullity of the stiffness matrix was large. It was also shown in 12 that round-off errors did not play a significant role in solving the linear system, i.e., the round-off error was also the same as when the finite element linear system was solved. An iterative algorithm was also given in [12]. Therefore, there exist nowadays efficient solvers to deal with singular or near singular linear systems.

- Even if it leads to singular linear systems, $\mathcal{F}_{\mathcal{X}}^{m, r}$ yields better results than $\mathcal{F}_{\mathcal{D}}^{m, r}$

4.1. A 1-dimensional numerical experiment. We consider the model problem

$$
\begin{aligned}
-u^{\prime \prime} & =f \text { in } \Omega=[0,1], \\
u \mid \partial \Omega & =0,
\end{aligned}
$$

where $f$ is such that the exact solution is

$$
u(x)=\sin (2 \pi x) .
$$

We first make all settings in our experiment explicit.

- $\mathcal{S}_{N}:=\left\{\mathcal{W}_{i}\right\}_{i=1}^{N}$ will be the standard $(1,1)$-partition of unity of FEM of linear precision.

- Tests with both random and uniformly spaced nodes at several widths $h=$ $1 / 4,1 / 8,1 / 16,1 / 32$ have been performed. In the former case, nodes were generated by adding a random perturbation of value $0.25 h$ to a uniform grid with $h$-spacing. Computed errors in the random distribution points case, correspond to averages over ten runs.

- $\mathcal{F}_{\mathcal{X}}^{1, r}$ and $\mathcal{F}_{\mathcal{D}}^{1, r}$ have been compared at equal algebraic precision ap $=2,3,4$. In $\mathcal{F}_{\mathcal{X}}^{1, r}$. ap $=1+r$ while $a p=r$ for $\mathcal{F}_{\mathcal{D}}^{1, r}$.

- An $(r+1)$-point Gaussian quadrature has been used at interior cells. Note that, at equal algebraic precision, less points are used for $\mathcal{F}_{\mathcal{X}}^{1, r}$.

- Two relative errors have been computed:

$$
e_{\text {rel }}:=\frac{\left\|\widehat{u}_{h}-u_{\text {exact }}\right\|_{L^{2}(\Omega)}}{\left\|u_{\text {exact }}\right\|_{L^{2}(\Omega)}}
$$

and

$$
e x_{r e l}:=\frac{\left\|\left(\widehat{u}_{h}-u_{\text {exact }}\right)_{x}\right\|_{L^{2}(\Omega)}}{\left\|\left(u_{\text {exact }}\right)_{x}\right\|_{L^{2}(\Omega)}} .
$$

In these tests, errors have been calculated using a 5-point Gaussian quadrature at interior cells. 
- In order to prevent numerical instabilities, polynomials in $\mathcal{P}_{i}^{r}$ must be normalized by a measure of the size of the grid at $x_{i}$. That is, the basis of $\mathcal{P}_{i}^{r}$ (or $Q_{i}$ in the $\mathcal{F}_{\mathcal{D}}^{1, r}$-scheme) must be written in the form

$$
\left\{1,\left(\frac{x-x_{i}}{h_{i}}\right), \ldots,\left(\frac{x-x_{i}}{h_{i}}\right)^{r}\right\} .
$$

- In all tests, linear systems have been solved with the standard solver of MATLAB (C)The MathWorks, without any additional precaution.

4.1.1. $p$ adaptivity. In the first test we have fixed a uniform grid with $h=0.125$, varying ap from 4 to 2 . Errors $e_{r e l}$ and $e x_{r e l}$ from computations are summarized in Tables 1 and 2 respectively.

TABLE 1. Error $e_{r e l}, h=0.125$, varying $a p$.

\begin{tabular}{|c|c|c|c|}
\hline$a p$ & 4 & 3 & 2 \\
\hline $\mathcal{F}_{\mathcal{X}}^{1, r}$ & $3.7229_{-6}$ & $1.2562_{-4}$ & $2.7428_{-3}$ \\
\hline $\mathcal{F}_{\mathcal{D}}^{1, r}$ & $4.8678_{-5}$ & $1.1703_{-3}$ & $1.2426_{-2}$ \\
\hline
\end{tabular}

TABLE 2. Error $e x_{r e l}, h=0.125$, varying $a p$.

\begin{tabular}{|c|c|c|c|}
\hline$r$ & 4 & 3 & 2 \\
\hline $\mathcal{F}_{\mathcal{X}}^{1, r}$ & $7.5158_{-5}$ & $1.5181_{-3}$ & $2.2906_{-2}$ \\
\hline $\mathcal{F}_{\mathcal{D}}^{1, r}$ & $8.9651_{-4}$ & $1.3084_{-2}$ & $8.0095_{-2}$ \\
\hline
\end{tabular}

4.1.2. $h$ adaptivity. In this test with random grids, we have fixed $a p=2$, varying $h$ from $1 / 4$ to $1 / 32$. Errors $e_{r e l}$ and $e x_{r e l}$ from computations are summarized in Tables 3 and 4 respectively.

TABLE 3. Error $e_{r e l}, a p=2$, varying $h$.

\begin{tabular}{|c|c|c|c|c|}
\hline$h$ & $1 / 32$ & $1 / 16$ & $1 / 8$ & $1 / 4$ \\
\hline $\mathcal{F}_{\mathcal{X}}^{1,1}$ & $5.4401_{-6}$ & $4.3487_{-5}$ & $3.4686_{-4}$ & $2.7428_{-3}$ \\
\hline $\mathcal{F}_{\mathcal{D}}^{1,2}$ & $2.1963_{-5}$ & $1.7785_{-4}$ & $1.4657_{-3}$ & $1.2426_{-2}$ \\
\hline
\end{tabular}

TABLE 4. Error $e x_{r e l}, a p=2$, varying $h$.

\begin{tabular}{|c|c|c|c|c|}
\hline$h$ & $1 / 32$ & $1 / 16$ & $1 / 8$ & $1 / 4$ \\
\hline $\mathcal{F}_{\mathcal{X}}^{1,1}$ & $3.5917_{-4}$ & $1.4364_{-3}$ & $5.7419_{-3}$ & $2.2906_{-2}$ \\
\hline $\mathcal{F}_{\mathcal{D}}^{1,2}$ & $1.4187_{-3}$ & $5.5980_{-3}$ & $2.1708_{-2}$ & $8.0095_{-2}$ \\
\hline
\end{tabular}


We may appreciate that the $\mathcal{F}_{\mathcal{X}}^{1, r}$-method has a more equal convergence rate than the $\mathcal{F}_{\mathcal{D}}^{1, r}$-method, but $\mathcal{F}_{\mathcal{X}}^{1, r}$ produces accurate solutions with one more digit of precision.

4.2. 2-dimensional numerical experiments. We consider $\Omega \subset \mathbb{R}^{2}$ and the weak formulation of a Poisson model problem

$$
\begin{aligned}
-\Delta u & =f \quad \text { in } \Omega, \\
u & =g \quad \text { on } \Gamma_{d} \subset \partial \Omega, \\
u_{n} & =\frac{\partial u}{\partial n}=q \quad \text { on } \Gamma_{n} \subset \partial \Omega .
\end{aligned}
$$

Adding Taylor polynomials at nodes $x_{j} \in \Gamma_{d}$ clearly presents a problem even if the partition of unity $\left\{\mathcal{W}_{i}\right\}$ satisfies the delta-Kroenecker condition $\mathcal{W}_{i}\left(x_{j}\right)=\delta_{i, j}$. Furthermore, the Taylor polynomials could improve interpolation at the boundary by using an appropriate treatment of Dirichlet data.

In the early 1970s, J. Nitsche developed a general approach for the treatment of essential boundary conditions where the shape functions do not have to fulfil the boundary conditions [10. In this work, we have used a non-stabilized Nitsche's method. Roughly speaking, we have considered the weak formulation:

Find $u \in \mathcal{F}_{h}$ such that

$$
\int_{\Omega} \nabla u \cdot \nabla v-\int_{\Gamma_{d}}\left[u_{n} v+u v_{n}\right]+\beta \int_{\Gamma_{d}} u v=\int_{\Omega} f v+\int_{\Gamma_{d}} g\left(\beta v-v_{n}\right)+\int_{\Gamma n} q v
$$

for all $v \in \mathcal{F}_{h}$.

We refer to [7] for a complete description of the method.

The settings for experiments are:

- The triangle mesh generator Easymesh (CBojan Niceno) was used in order to obtain triangulations using data nodes at the boundary. In $\Omega=[0,1] \times$ $[0,1]$, three meshes have been generated for uniform data in $\partial \Omega$ at several mesh widths $h=1 / 4,1 / 8,1 / 16$.

- Four relative errors: $e_{r e l}, e x_{r e l}, e y_{r e l}$ and $e_{\infty, r e l}$ have been calculated using a 7-point quadrature formula at interior cells.

- Errors for the standard linear FEM $\mathcal{F}^{1,0}$ and quadratic FEM $\mathcal{F}^{2,0}$ have been computed for the sake of comparison.

- We write $\mathcal{F}^{m, 0}(s), \mathcal{F}^{m, r}(s, t)$ to indicate that an $s$-point quadrature formula has been used at interior cells and a $t$-point quadrature formula has been used at boundary cells.

4.2.1. Model 1. We consider the problem

$$
\begin{aligned}
u_{x x}+u_{y y} & =f(x, y) \quad \text { in } \Omega=[0,1] \times[0,1], \\
u \mid \partial \Omega & =0
\end{aligned}
$$

and choose $f$ such that the exact solution is

$$
u(x, y)=\arctan \left(100\left(\frac{x+y}{\sqrt{2}}\right)\left(x-x^{2}\right)\left(y-y^{2}\right)\right) .
$$

Errors for several Galerkin spaces $\mathcal{F}_{h}$ are summarized in Tables 5, 6, 7, 8, 9 and 10. The results clearly show the superiority of $\mathcal{F}_{\mathcal{X}}^{1, r}$ (it is also useful to compare our results with those in [7]). 
TABLE 5. Error $e x_{r e l}, a p=2$, varying $h$.

\begin{tabular}{|c|c|c|c|c|}
\hline$h$ & $1 / 32$ & $1 / 16$ & $1 / 8$ & $1 / 4$ \\
\hline $\mathcal{F}_{\mathcal{X}}^{1,1}$ & $3.5917_{-4}$ & $1.4364_{-3}$ & $5.7419_{-3}$ & $2.2906_{-2}$ \\
\hline $\mathcal{F}_{\mathcal{D}}^{1,2}$ & $1.4187_{-3}$ & $5.5980_{-3}$ & $2.1708_{-2}$ & $8.0095_{-2}$ \\
\hline
\end{tabular}

TABle 6. Model $1, V_{h}=\mathcal{F}^{1,0}(1)$.

\begin{tabular}{|c|c|c|c|c|}
\hline Dof & $e_{\text {rel }}$ & $e x_{\text {rel }}$ & eyrel & $e_{\infty, \text { rel }}$ \\
\hline 60 & $6.9342_{-2}$ & $3.2427_{-1}$ & $3.1235_{-1}$ & $1.2834_{-1}$ \\
\hline 267 & $1.9699_{-2}$ & $1.6170_{-1}$ & $1.5594_{-1}$ & $4.2933_{-2}$ \\
\hline 1131 & $5.3033_{-3}$ & $7.9956_{-2}$ & $7.9705_{-2}$ & $1.2670_{-2}$ \\
\hline
\end{tabular}

TABLE 7. Model $1, V_{h}=\mathcal{F}^{2,0}(3)$.

\begin{tabular}{|c|c|c|c|c|}
\hline Dof & $e_{\text {rel }}$ & ex rel & eyrel & $e_{\infty, \text { rel }}$ \\
\hline 269 & $1.3409_{-2}$ & $4.7132_{-2}$ & $4.5140_{-2}$ & $2.8684_{-2}$ \\
\hline 1129 & $1.3842_{-3}$ & $1.3368_{-2}$ & $1.2610_{-2}$ & $4.1615_{-3}$ \\
\hline 4649 & $1.1739_{-4}$ & $3.7489_{-3}$ & $3.6917_{-3}$ & $8.3689_{-4}$ \\
\hline
\end{tabular}

TABLE 8. Model $1, V_{h}=\mathcal{F}_{\mathcal{X}}^{1,1}(3,2)$.

\begin{tabular}{|c|c|c|c|c|}
\hline Dof & $e_{\text {rel }}$ & $e x_{\text {rel }}$ & eyrel & $e_{\infty, \text { rel }}$ \\
\hline 244 & $8.0937_{-3}$ & $4.1408_{-2}$ & $4.1401_{-2}$ & $1.8508_{-2}$ \\
\hline 929 & $9.2963_{-4}$ & $1.1114_{-2}$ & $1.0462_{-2}$ & $2.5599_{-3}$ \\
\hline 3649 & $8.9332_{-5}$ & $3.1773_{-3}$ & $3.1075_{-3}$ & $4.4461_{-4}$ \\
\hline
\end{tabular}

TABLE 9. Model $1, V_{h}=\mathcal{F}_{\mathcal{D}}^{1,2}(3,2)$.

\begin{tabular}{|c|c|c|c|c|}
\hline Dof & $e_{\text {rel }}$ & $e x_{\text {rel }}$ & ey rel & $e_{\infty, \text { rel }}$ \\
\hline 333 & $2.3245_{-2}$ & $1.4096_{-1}$ & $1.3446_{-1}$ & $4.3396_{-2}$ \\
\hline 1196 & $2.6080_{-3}$ & $4.0184_{-2}$ & $3.7808_{-2}$ & $7.2470_{-3}$ \\
\hline 4908 & $3.4064_{-4}$ & $1.2078_{-2}$ & $1.1671_{-2}$ & $1.7633_{-3}$ \\
\hline
\end{tabular}


TABLE 10. Model $1, V_{h}=\mathcal{F}_{\mathcal{X}}^{1,2}(7,5)$.

\begin{tabular}{|c|c|c|c|c|}
\hline Dof & $e_{\text {rel }}$ & $e x_{r e l}$ & $e y_{\text {rel }}$ & $e_{\infty, \text { rel }}$ \\
\hline 520 & $1.006_{-3}$ & $8.0796_{-3}$ & $7.5917_{-3}$ & $2.7966_{-3}$ \\
\hline 1858 & $1.1419_{-4}$ & $1.8740_{-3}$ & $1.6288_{-3}$ & $4.7398_{-4}$ \\
\hline 7426 & $7.7560_{-6}$ & $2.3461_{-4}$ & $2.3087_{-4}$ & $4.0117_{-5}$ \\
\hline
\end{tabular}

4.2.2. Model 2. This test example is a Poisson equation with a harmonic source. Neumann boundary conditions are imposed:

$$
\begin{aligned}
-\left(u_{x x}+u_{y y}\right)+u & =\left(8 \pi^{2}+1\right) \cos (2 \pi x) \sin (2 \pi y) \quad \text { in } \Omega=[0,1] \times[0,1], \\
u_{n} \mid \partial \Omega & =u_{s, n},
\end{aligned}
$$

where the exact solution is

$$
u_{s}(x, y)=\cos (2 \pi x) \sin (2 \pi y) .
$$

Results for $\mathcal{F}_{\mathcal{X}}^{1,1}$ and $\mathcal{F}^{2,0}$ are summarized in Tables 11 and 12 .

TABLE 11 . Model $1, V_{h}=\mathcal{F}_{\mathcal{D}}^{1,3}(7,5)$.

\begin{tabular}{|c|c|c|c|c|}
\hline Dof & $e_{\text {rel }}$ & $e x_{r e l}$ & $e y_{r e l}$ & $e_{\infty, r e l}$ \\
\hline 704 & $2.8760_{-3}$ & $2.3741_{-2}$ & $2.2613_{-2}$ & $6.5160_{-3}$ \\
\hline 2520 & $2.8020_{-4}$ & $4.3350_{-3}$ & $3.8011_{-3}$ & $9.4980_{-4}$ \\
\hline 9944 & $4.1614_{-5}$ & $1.0973_{-3}$ & $1.0740_{-3}$ & $2.8198_{-4}$ \\
\hline
\end{tabular}

TABLE 12. Model $2, V_{h}=\mathcal{F}^{2,0}(3)$.

\begin{tabular}{|c|c|c|c|c|}
\hline Dof & $e_{\text {rel }}$ & $e x_{r e l}$ & $e y_{\text {rel }}$ & $e_{\infty, \text { rel }}$ \\
\hline 333 & $4.2717_{-3}$ & $3.3197_{-2}$ & $3.4645_{-2}$ & $7.6092_{-3}$ \\
\hline 1257 & $5.3919_{-4}$ & $8.6610_{-3}$ & $8.2048_{-3}$ & $1.1223_{-3}$ \\
\hline 4905 & $6.4327_{-5}$ & $2.2884_{-3}$ & $1.8482_{-3}$ & $1.2913_{-4}$ \\
\hline
\end{tabular}

TABLE 13. Model $2, V_{h}=\mathcal{F}_{\mathcal{X}}^{1,1}(3,2)$.

\begin{tabular}{|c|c|c|c|c|}
\hline Dof & $e_{\text {rel }}$ & $e x_{\text {rel }}$ & eyrel & $e_{\infty, \text { rel }}$ \\
\hline 276 & $5.0433_{-3}$ & $3.5308_{-2}$ & $3.6448_{-2}$ & $9.2298_{-3}$ \\
\hline 993 & $5.7019_{-4}$ & $8.9559_{-3}$ & $8.3591_{-3}$ & $1.1162_{-3}$ \\
\hline 3777 & $6.5773_{-5}$ & $3.3225_{-3}$ & $1.8579_{-3}$ & $1.3052_{-4}$ \\
\hline
\end{tabular}


Remark 4.1. It is clear that $\mathcal{F}_{\mathcal{X}}^{1,1} \subset \mathcal{F}^{2,0}$, but $\mathcal{F}_{\mathcal{X}}^{1,1}(3,2)$ produces in general equal or better results than $\mathcal{F}^{2,0}(3)$ with smallest DOF. This is an amazing fact in the context of Galerkin approximation.

It appears that this methodology has a number of useful features. Even if the stiffness matrix is singular, the method seems to be robust. Dirichlet boundary conditions are easily handled with Nitsche's method.

\section{REFERENCES}

[1] S.C. Brenner and L.R. Scott, The Mathematical Theory of Finite Elements Methods, Springer-Verlag, New York, 1994. MR.1278258 (95f:65001)

[2] C. A. M. Duarte, H-p Clouds - an h-p Meshless Method, Ph.D. Thesis, TICAM, The University of Texas at Austin, 1995.

[3] C. A. M. Duarte and J. T. Oden, Hp clouds - an hp meshless method to solve boundary value problems, Technical Report 59-05, TICAM, The University of Texas at Austin, 1995.

[4] C. A. M. Duarte and J. T. Oden, H-p Clouds-an h-p Meshless Method, Numer. Methods for Partial Diff. Eq. 12 (1996), pp. 673-705. MR1419770(98a:65163)

[5] R. G. Durán, On polynomial approximation in Sobolev spaces, SIAM J. Numer. Anal. 20 (1983), pp. 985-988. MR714693 (85e:42010)

[6] T. Dupont and R. Scott, Polynomial Approximation of Functions in Sobolev Spaces, Math. of Comp. 34/150 (1980), pp. 441-463. MR559195 (81h:65014)

[7] M. Griebel and M. A. Schweitzer, A particle partition of unity method, V, Boundary conditions, Geometric Analysis and Nonlinear Partial Differential Equations, S. Hildebrand and H. Karcher, eds., pp. 517-540, 2003. MR2008354

[8] A. Guessab, O. Nouisser and G. Schmeisser, Multivariate approximation by a combination of modified Taylor polynomials, J. Comput. Appl. Math. 196 (2006), 162-179. MR.2241582 (2007a:41057)

[9] S. R. Idelsohn, E. Oñate, N. Calvo and F. Del Pin, The meshless finite element method, Int. J. Num. Meth. Engrg. 58 (2003), pp. 893-912. MR2010710

[10] J. Nitsche, Über ein Variationaprinzip zur Lösing Dirichlet-Problemen bei Verwerdung von Teilräumen, die keinen Randbedingungen unterworfen sind, Abh. Math. Univ. Hamburg 36 (1970/71), pp. 9-15. MR0341903(49:6649)

[11] J. T. Oden, C. A. M. Duarte and O. C. Zienkiewicz, A new cloud-based hp finite element method, Comput. Methods Appl. Mech. Engrg. 153 (1998), pp. 117-126. MR1606117 (99c:73081)

[12] T. Strouboulis, I. Babuška and K. Copps, The design and analysis of the generalized finite element method, Comput. Methods Appl. Mech. Engrg. 181 (2001), pp. 43-69. MR.1734667 (2000h:74077)

[13] R. Verfürth, Error estimates for some quasi-interpolation operators, RAIRO Modél. Anal. Numér. 33 (1999), pp. 695-713. MR1726480 (2001a:65149)

[14] R. Verfürth, A note on polynomial approximation in Sobolev spaces, RAIRO Modél. Anal. Numér. 33 (1999), pp. 715-719. MR1726481 (2000h:41016)

[15] H. Xuli, Multi-node higher order expansions of a functions, J. Approx. Theory 124 (2003), pp. 242-253. MR2016674 (2004k:41059)

[16] C. Zuppa, Jackson-type inequalities for $h-p$ clouds and error estimates, Comput. Methods Appl. Mech. Engrg. 194 (2005), pp. 1875-1887. MR2121320 (2005j:65152)

Departamento de Matemáticas, Universidad Nacional de San Luis, San Luis 5700, ARgENTINA

E-mail address: zuppa@unsl.edu.ar 